\title{
A common source outbreak of Acinetobacter pulmonary infections traced to Wright respirometers
}

\author{
Burke A. Cunha \\ M.D. \\ JOHN GRACEWSKI \\ B.S.

\begin{abstract}
Summary
Over a 30-day period, Acinetobacter calcoaceticus var. antiratus was the responsible pathogen for hospital-acquired pneumonia in 10 patients, and resulted in the colonization of the upper respiratory tract in an additional 9 patients. Wright respirometers contaminated by this organism were shown to be the common source for the outbreak as indicated by the recovery of a single serotype $(8 \mathrm{~J})$, the inability to recover Acinetobacter from any other environmental source, and the demonstration that moisturized Wright respirometers are capable of 'aerosolizing' fluids containing Acinetobacter.
\end{abstract}

\author{
JOSEPH J. İLIMEK \\ M.D. \\ James C. McLaughlin \\ Ph.D.
}

\author{
RICHARD QUINTILIANI \\ M.D.
}

Department of Medicine, and Department of Pathology, Hartford Hospital, Hartford, Connecticut

\section{Introduction}

Acinetobacter calcoaceticus var. anitratus is a relatively frequent cause of upper respiratory tract colonization in hospital patients (Irving and Herrick, 1967; Inclan et al., 1965; Green, Johnson and Shively, 1960; Gardner et al., 1970; Ramphal and Kluge, 1979). Acinetobacter is a hydrophilic organism, and respiratory infections due to this organism have been associated with fine particle nebulizers and humidifiers. These outbreaks have always involved multiple Acinetobacter serotypes (Kelsen et al., 1977; Smith and Massanan, 1977). In this communication, the clinical and epidemiological features are reported of a large outbreak of respiratory tract colonization and infection, in patients in hospital, due to a single Acinetobacter serotype (8J). Contaminated Wright respirometers were found to be the common source of the outbreak.

Requests for reprints: Dr Burke A. Cunha, Infectious Disease Division, Department of Medicine, Nassau Hospital, Mineola, NY 11501, U.S.A.
Materials and methods

Patients

Hartford Hospital, Connecticut, is a 1000-bed, university affiliated, teaching hospital with 6 intensive care units. The outbreak consisted of 10 patients who developed evidence of Acinetobacter pneumonia, and an additional 9 patients who were colonized over a period of $\mathbf{3 0}$ days in $\mathbf{4}$ intensive care units. Patients with a lobar pulmonary infiltrate on chest film, fever, leucocytosis and a purulent sputum containing Acinetobacter as the sole or predominant organism were considered to have Acinetobacter pneumonia.

\section{Microbiology}

Sputum specimens were collected by nasotracheal aspiration, examined in the laboratory by Gram stain and cultured on MacConkey and $5 \%$ sheep blood agar plates. Swabs of respiratory equipment were transported to the laboratory in Amies transport medium and plated on the above media. After overnight incubation in air with $5 \% \mathrm{CO}_{2}$ added, the agar plates were examined. When 6 or more colonies of presumptive Gram-negative organisms were observed, a representative colony of each type was inoculated into an API $20 \mathrm{E}$ biochemical strip for identification. The antibiotic susceptibility of Acinetobacter to ampicillin, cephalothin, gentamicin, kanamycin, tobramycin, co-trimoxazole, minocycline and chloramphenicol was determined by the Bauer-Kirby method (National Committee for Laboratory Standards, Los Angeles, 1976). Isolates identified as Acinetobacter were serotyped in the Analytical Bacteriology Branch at the Center for Disease Control. 


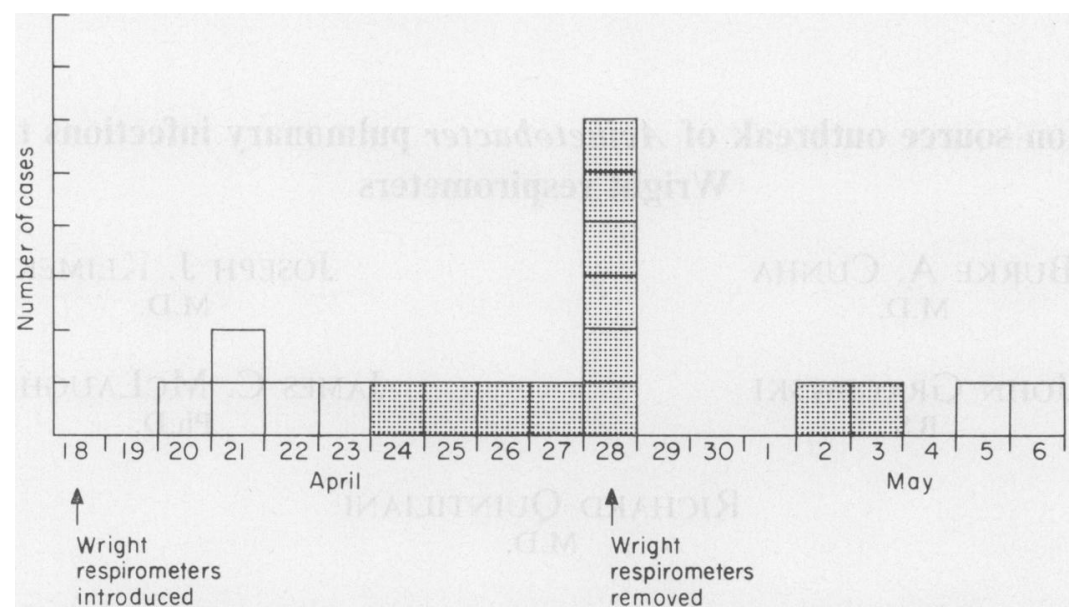

FIG. 1. Acinetobacter infections and colonizations at Hartford Hospital, Connecticut, from April to May 1977. $\square$, one case, colonization; 㐘, one case infection.

\section{Epidemiology}

Description of the outbreak

The usual incidence of Acinetobacter isolates from the sputum at Hartford Hospital is 235.8 cases $/ 10^{5}$ patients or an average of $7 \cdot 4$ isolates per month. During routine epidemiological surveillance in April, an abrupt and unexplained increase in the recovery of Acinetobacter from sputum cultures was noted with 732.9 cases $/ 10^{5}$ or 19 cases occurring during the month of the outbreak (Fig. 1).

During this outbreak, 10 patients developed Acinetobacter pneumonia approximately 8 days (range 6-10 days) after the introduction of Wright respirometers.

Acinetobacter was recovered from purulent sputum approximately 13 days (range 10-19 days) after the introduction of the respirometers. The fever peaked on the 14th day (range 10-19 days), and ranged from $100 \cdot 6^{\circ}$ to $106 \cdot 4^{\circ} \mathrm{F}$. A patchy infiltrate appeared on chest X-ray about 10 days (range 8-12 days) after the introduction of the respirometers. The initial radiological abnormality detected in all patients was that of a diffuse infiltrate in one or both of the lower lobes. All patients eventually developed spread to the contralateral lower lobe, and 2 of the patients also developed upper lobe involvement. Additionally, 2 patients developed diffuse bronchopneumonia during the course of their infection. No patient developed a pleural effusion or sign of consolidation by X-ray or physical examination. White blood cell counts also peaked on day 13 (range 8-25 days) of the outbreak. Peak WBC counts averaged $19.8 \times 10^{9} / 1$ (range $15.4 \times 10^{9} / 1-2.6 \times 10^{9} \mathrm{l} /$ ) with the exception of a pre-leukaemic patient who remained leucopenic while in hospital. Shaking chills or chest pain were not present during the illness of any patient. Acinetobacter was not recovered from the blood of any patient during the outbreak.

\section{Investigation}

When the occurrence of the outbreak was recog. 9 nized, extensive sampling of equipment and persog- $\overrightarrow{0}$ nel was quickly carried out. Nasal and skin cultukes of medical and para-medical personnel in tei intensive care units were obtained, as were culturso of the suction apparatus, respirators, respirator tubing, respirator air effluent, and intravenous poly-a ethylene catheters.

No organisms were recovered from any of the $\stackrel{\square}{\Rightarrow}$ environmental sources cultured, but Acinetobacter was recovered from patients and respirometers in the intensive care units. Further investigation revealed that the Department of Respiratory Therapy had removed the Bourns* respirometers for repairs and음 substituted 5 Wright $\dagger$ respirometers. Because the number of respirometers available as interim re- 3 placements was limited, the respirometers were? shared by 4 intensive care units.

\section{Control measures}

The initial steps to control the outbreak included restricting the use of each Wright respirometer to $\mathrm{a}$. single intensive care unit thereby eliminating the of inter-intensive care unit sharing of equipment. Then importance of hand washing precautions was N

* The Bourns LS 75 respirometer has no moving parts, andర operates on the vortex principle. A flow sensor in the device detects vibrations in the airstream by an ultrasonic beam $ᄃ$ and, via a miniature computer, converts wave motion into volume flow rates.

$\dagger$ The Wright respirometer utilizes a small rotor placed in 0 the airstream of the device. The small air turbine is calibrated so that its revs/sec correspond to volume flow rates. 
emphasized to intensive care personnel, and strict enforcement of careful hand washing technique was observed. Because the cleaning of Wright respirometers with alcohol is technically not possible, it was decided to modify respirometer usage in an attempt to minimize the exposure of each patient to respirometers. This was accomplished by obtaining volume measurements with the respirometers placed at the most distal portion of the respirator tubing instead of interposing the respirometer between the patient and the endotracheal or tracheostomy tubing. This manoeuvre minimized the possibility of 'aerosolizing' the organism into the bronchioles of patients not previously colonized or infected by Acinetobacter.

\section{Respirometer investigation}

Laboratory tests were designed to simulate the high moisture content of the air exhaled by patients while on a respirator. Gas-sterilized Wright respirometers were exposed to sterile saline via a sterile nebulizer attached to the same type of volume respirator used in the intensive care units. Subsequently, sterilized Wright respirometers were exposed to a suspension of Acinetobacter via a sterile nebulizer attached to a volume respirator. Effluent air samples were obtained for culture using the Andersen air sampler.

\section{Results \\ Patients}

All patients colonized or infected had severe underlying disease requiring assisted ventilation in an intensive care unit. Because of the presence of unexplained fever, all patients received empiric antibiotic therapy before the diagnosis of Acinetobacter pneumonia. Two patients received cephazolin and 4 received both cephazolin and gentamicin for 1 to 4 days before the correct diagnosis was made. When the diagnosis of Acinetobacter pneumonia was established, patients with pneumonia were treated with either tobramycin, $80 \mathrm{mg}$ i.v. every $8 \mathrm{hr}$, or minocycline, $150 \mathrm{mg}$ intravenously every $12 \mathrm{hr}$, for 4 to 14 days based on antibiotic disc susceptibility tests.

Although no patient died directly as a result of Acinetobacter pneumonia it is likely that the organism contributed to the deaths of 3 patients. The remaining patients with pneumonia became afebrile after approximately 6 days (range 2-12 days) of antimicrobial therapy, and were discharged without Acinetobacter in the sputum. Sputum cultures of patients colonized by Acinetobacter eventually became negative after 6-18 days without antimicrobial therapy.

\section{Microbiology}

Sensitivity testing by disc diffusion technique demonstrated uniform resistance of Acinetobacter to ampicillin, cephalosporins, gentamicin, and chloramphenicol. Some strains were sensitive to kanamycin or to tobramycin. The only antibiotics tested to which all the Acinetobacter strains tested were consistently sensitive were minocycline and co-trimoxazole. Acinetobacter recovered from the respirometers and colonized patients demonstrated the same sensitivity patterns recovered from infected patients except for considerable variation in sensitivity to kanamycin and tobramycin by disc testing. Throughout the outbreak, Acinetobacter was recovered only from sputum and the Wright respirometers. The common source nature of the outbreak is further supported by finding only one serotype (8J) involved in the entire outbreak.

\section{Epidemiology}

Recovery rate of the organism from sputum began to decrease, and no new cases occurred 6 days after the institution of control measures. At this time the repaired Bourns respirometers were returned, and the Wright respirometers were removed from service. An additional 18 days were required fully to eliminate the carriage of Acinetobacter from the sputum of the colonized patients. Retrospectively, the outbreak lasted 30 days from the introduction of the Wright respirometers to the elimination of respiratory carriage of the organism.

\section{Respirometer investigation}

Studies were conducted to determine if moisturized Wright respirometers, contaminated with Acinetobacter, could be responsible for the transmission of the organism by 'aerosolization'. Air samples obtained through Wright respirometers exposed to Acinetobacter as described in materials and methods, consistently revealed colonies of Acinetobacter too numerous to count. Air samples obtained through Wright respirometers exposed to sterile saline were either sterile or yielded air contaminants only.

\section{Discussion}

A large outbreak of respiratory tract infection and colonization due to Acinetobacter occurred in 4 intensive care units over a 30-day period.

Patients who developed Acinetobacter pneumonia were all pre-treated with antibiotics, had serious underlying diseases, and required assisted ventilation in an intensive care setting.

Acinetobacter pneumonia was characterized by leucocytosis, fever, purulent sputum, and diffuse lower lobe infiltrates without consolidation or effusion. Blood cultures were negative in all patients. 
These findings are consistent with previously published reports indicating that bacteraemia is not a characteristic feature of the disease (Glew, Moellering and Kunz, 1977; Glick et al., 1959). Appropriate antibiotic selection, based on sensitivity disc testing, included minocycline, tobramycin, and cotrimoxazole (Maderazo et al., 1975). Patients with pneumonia were treated with either parenteral minocycline or tobramycin.

Studies performed during the outbreak demonstrate that moisturized Wright respirometers were capable of aerosolizing Acinetobacter. Although these studies did not involve patients the data suggest that this may explain the role of the respirometers in the patient-to-patient transmission of Acinetobactef in intensive care units where Wright respirometers are directly attached to the patient's endotracheal or tracheostomy tubing.

Control measures included rigorous hand washing techniques by intensive care personnel, restricting the use of individual Wright respirometers to only one intensive care unit, and the taking of volume measurements with the respirometer from the ventilator tubing instead of directly from the patient's endotracheal or tracheostomy tube. Since the elimination of the patients' colonization by the organism, and the re-introduction of the Bourns respirometers, the recovery of Acinetobacter from sputum has returned to pre-outbreak levels.

Serotyping confirmed the initial clinical and epidemiological impression that the Wright respirometers containing Acinetobacter were solely responsible for the outbreak.

\section{Acknowledgments}

The authors wish to express their appreciation to Ms Bertie Pittman of the Analytical Bacteriology Branch, Bureau of Laboratories, Center for Disease Control, Atlanta, Georgia, for serotyping, Ms Elizabeth Ajemian and Ms
Barbara Klemas of the Section of Epidemiology, and the Department of Respiratory Therapy and the Technologist of the Division of Microbiology for their help and con operation.

\section{References}

Gardner, P., Griffin, W.B., SWARtz, M.N. \& Kunz, L (1970) Nonfermentative Gram-negative bacilli of nosocomial interest. American Journal of Medicine, 48, 735.

Glew, R.H., Moellering, R.C. \& Kunz, L.J. (1977) Infec tions with Acinetobacter calcoaceticus (Herellea vaginicola) Clinical and laboratory studies. Medicine, 56, 79.

Glick, L.M., Moran, G.P., Coleman, J.M. \& O'Brien, G.Fొ़ (1959) Lobar pneumonia with bacteremia caused b5 Bacterium anitratum. American Journal of Medicine, 27 183.

GreEn, G.S., Johnson, R.H. \& Shively, J.A. (1960) Mimeae Opportunistic pathogens. Journal of the American Medicat Association, 174, 163.

InClan, A.P., Massey, L.C., CrooK, B.G. \& Bell, J.S. (196\$) Organisms of the tribe Mimeae: Incidence of isolation an 4 clinical correlation at the City of Memphis Hospitals
Southern Medical Journal, 58, 1261 .

Irving, W.R. \& Herrick, W.C. (1967) The bacteria Mims Herellea: Isolation and clinical significance in a generab hospital. American Journal of Clinical Pathology, 47, 729.

Kelsen, S.G., MCGuCKIn, M., Kelsen, D.P. \& Chernicko N.S. (1977) Airborne contamination of fine particle nebulizers. Journal of the American Medical Association 237, 2311.

Maderazo, E.G., Quintiliani, R., Tilton, R.C., BartletT R., JOYCE, H.C. \& ANDRIOLE, V.T. (1975) Activity of minocycline against Acinetobacter calcoaceticus $\operatorname{rar}$ anitratus. Antimicrobial Agents and Chemotherapy, 8, 59

NATIONAL COMmitTeE FOR LABORATORY STANDARDS (19 6.9 Performance Standards for Antimicrobial Disc Susceñiti bility Tests, as used in Clinical Laboratories. Revise Tentative Standard, Los Angeles.

RAMPHAL, R. \& KLUGE, R.M. (1979) Acinetobacter calcō̄ aceticus variety anitratus: an increasing nosocomiaf problem. American Journal of the Medical Sciences, $277 \mathrm{~g}$ 57.

Robinson, R.G., Garrison, R.G. \& Brown, R.W. (1964 $\overrightarrow{\bar{\delta}}$ Evaluation of the clinical significance of the genus Herellea. Annals of Internal Medicine, 60, 19.

Smith, P.W. \& Massanan, R.M. (1977) Room humidifier as the source of Acinetobacter infections. Journal of the American Medical Association, 237, 2311. 